\title{
Comparative validation of assessment criteria for Crohn-like lymphoid reaction in colorectal carcinoma
}

\author{
Jung Ho Kim, ${ }^{1}$ Kyung-Ju Kim, ${ }^{2}$ Jeong Mo Bae, ${ }^{2}$ Ye-Young Rhee, ${ }^{2}$ Nam-Yun Cho, ${ }^{3}$ \\ Hye Seung Lee, ${ }^{4}$ Gyeong Hoon Kang ${ }^{2,3}$
}

- Additional material is published online only. To view please visit the journal online (http://dx.doi.org/10.1136/ jclinpath-2014-202603).

${ }^{1}$ Department of Pathology, SMG-SNU Boramae Medical Centre, Seoul, Korea 2Department of Pathology, Seoul National University College of Medicine, Seoul, Korea

${ }^{3}$ Laboratory of Epigenetics, Cancer Research Institute, Seoul National University College of Medicine, Seoul, Korea

${ }^{4}$ Department of Pathology, Seoul National University Bundang Hospital, Seongnam, Korea

\section{Correspondence to} Professor Gyeong Hoon Kang, Department of Pathology, Seoul National University College of Medicine, 103 Daehak-ro, Jongno-gu, Seoul 110-799, Korea; ghkang@snu.ac.kr

Received 5 August 2014 Revised 19 September 2014 Accepted 30 September 2014 Published Online First 16 October 2014

\begin{abstract}
Aims Crohn-like lymphoid reaction (CLR) in colorectal carcinoma (CRC) is associated with a favourable prognosis and microsatellite instability-high (MSI-H) status. However, there is a lack of consensus on optimal criteria for CLR assessment. The aim of this study was to comparatively validate traditional and novel assessment criteria for CLR.
\end{abstract}

Methods CLR status in $212 \mathrm{MSI}-\mathrm{H}$ CRCs was assessed independently by two pathologists using three different criteria: (1) traditional semiquantitative criteria (GrahamAppelman criteria), (2) the largest lymphoid aggregate (LA) size-based criteria (Ueno criteria) and (3) LA density-based criteria (Väyrynen-Mäkinen criteria). Results Among the three criteria, the VäyrynenMäkinen criteria-based CLR assessment showed the best interobserver agreement ( $\kappa$ value, 0.71; intraclass correlation coefficient, 0.76). Pathologically, intense CLR (grade 2) by Graham-Appelman criteria, active CLR (largest LA size $\geq 1 \mathrm{~mm}$ ) by Ueno criteria and highdensity CLR ( $\geq 0.38$ LAs $/ \mathrm{mm}$ ) by Väyrynen-Mäkinen criteria significantly correlated with an early cancer stage (stage I/II). In Kaplan-Meier analysis, both CLR statuses determined by Ueno criteria and Väyrynen-Mäkinen criteria were associated with significant differences in disease-free survival in MSI-H CRC patients ( $p=0.005$ and $p=0.001$, respectively). In multivariable analysis, both active CLR and high-density CLR proved to be independent favourable prognostic factors in MSI-H CRC ( $H R, 0.47 ; 95 \% \mathrm{Cl} 0.24$ to 0.9 for active CLR and HR, $0.5 ; 95 \% \mathrm{Cl} 0.28$ to 0.89 for high-density (LR).

Conclusions Our study confirms that the two recently suggested criteria (Ueno criteria and Väyrynen-Mäkinen criteria) for CLR assessment are fairly reproducible methods and can serve as superior prognosticators in CRC.

\section{INTRODUCTION}

Crohn-like lymphoid reaction (CLR) consists of nodular lymphoid aggregates (LA) with or without germinal centres around areas of colorectal carcinoma (CRC), and is distributed in the deep portion of the bowel wall beyond invasive fronts of CRC, mainly in the muscularis propria and pericolic adipose tissue. ${ }^{1}$ Because the transmural appearance of LAs is reminiscent of the histological feature of Crohn's disease, the term 'Crohn-like' is used. CLR has been regarded as a type of host immune response against tumours, and therefore, it should be distinguished from normal physiological lymphoid structures, such as mucosa-associated lymphoid tissue and pericolic subserosal lymph nodes. Consistent with the expected functional relevance of CLR as an antitumour immune response, CLR has been identified as a histological prognosticator indicating improved survival in patients with CRC. $^{1-5}$

Since the first description and assessment of CLR as a prognostic factor in CRC by Graham and Appelman, ${ }^{1}$ a few histological assessment systems for CLR have been suggested. Graham and Appelman evaluated CLR using a semiquantitative three-tier grading method: absent (grade 0), no or one single LA in all tumour sections; mild (grade 1), occasional LAs with rare or absent germinal centres; intense (grade 2), numerous LAs with frequent germinal centres. Additionally, two novel assessment systems for CLR were recently reported by the Ueno group and the Väyrynen-Mäkinen group. Ueno et $a l^{4}$ suggested that the maximum diameter of LA in CLR was associated with prognostic significance in CRC and divided CRCs into two prognostic subgroups with active (large LA size) or inactive (small LA size) CLR using a cut-off diameter of $1 \mathrm{~mm}$. Alternatively, the density of CLR at the invasive margin of the tumour was suggested to be a significant prognostic factor in CRC by the Väyrynen-Mäkinen group. ${ }^{5}$ Although all these assessment criteria can be easy and useful methods for histological assessment of CLR, we sought to determine which assessment criterion has the most powerful prognostic value in CRC.

CLR is closely associated with microsatellite instability-high (MSI-H) in CRC. ${ }^{6-8}$ Thus, it is possible that the prognostic impact of CLR might be based mainly on MSI-H because MSI-H is generally considered to be a favourable prognostic factor in CRC. ${ }^{10}$ Therefore, in our present study, to minimise the prognostic effect of MSI-H and to clarify the prognostic value of CLR itself, the prognostic implications of CLR assessment criteria were analysed in a large series of MSI-H CRC. Through this analysis, we expected to clearly compare the prognostic values of CLR assessment criteria in CRC, independent of MSI-H status.

\section{MATERIALS AND METHODS \\ Case selection}

Totally, 212 MSI-H CRCs were retrospectively collected from the pathology archive of our institutions. All of the samples were formalin-fixed, paraffin-embedded (FFPE) tissues taken from resection specimens submitted for pathological diagnosis, and originated from patients with CRC who underwent curative surgical treatment between January 2004 and December 2008. During this time, MSI analysis was performed on 2957 consecutive series of resected CRCs by the molecular pathology laboratory of our institutions, and 
among them, 237 samples were diagnosed as MSI-H. Of these, 25 samples were excluded due to carcinoma in situ (pTis) or an inadequate quality of tissue sections for CLR evaluation, and finally, 212 samples were included in our present study. In the MSI analysis, the Bethesda guideline-recommended markers (BAT-25, BAT-26, D5S346, D17S250 and D2S123) were used, and MSI-H was determined when two or more markers showed instability in tumour DNA. ${ }^{11}$ This study received institutional review board approval (SNUH IRB No. H-1203-072-402).

\section{Histopathological assessment of CLR}

Histological assessment of CLR in 212 MSI-H CRCs was performed independently by two pathologists (JHK and K-JK), who were blinded to the clinicopathological and molecular data of the cases, using the three assessment criteria introduced above. As a pre-evaluation process, all representative tumour section slides (2 or 3 sections per case) of 212 MSI-H CRCs stained with H\&E were scanned using an automated scanner system (ScanScope XT; Aperio Technologies, Vista, California, USA). Subsequently, diameter and density of LAs were measured once using the ruler tool of digital image analyser software (ImageScope; Aperio Technologies). Discordant results among the two pathologists were reviewed and discussed, and a consensus was reached. The detailed evaluation criteria were as follows:

- Traditional semiquantitative three-tier grading criteria (Graham-Appelman criteria): absent (grade 0; figure 1A), no or one single LA in all tumour sections; mild (grade 1; figure 1B), occasional LAs with rare or absent germinal centres; intense (grade 2; figure 1C), numerous LAs with frequent germinal centres. ${ }^{1}$

- The largest LA size-based assessment criteria (Ueno criteria): active, the maximum diameter of the largest LA $\geq 1 \mathrm{~mm}$ (figure 1D); inactive, the maximum diameter of the largest LA $<1 \mathrm{~mm}$ (figure 1E). ${ }^{4}$

- LA density-based assessment criteria (Väyrynen-Mäkinen criteria): LA density was calculated as the number of CLR LAs/ the length of the invasive front of the tumour. All the study samples were dichotomously categorised into a high-density group (figure $1 \mathrm{~F}$ ) or low-density group (figure $1 \mathrm{G}$ ) using a cut-off point of $0.38 \mathrm{LAs} / \mathrm{mm}$ as suggested by Vayrynen et al. ${ }^{5}$

\section{Clinicopathological data}

Clinicopathological data of 212 MSI-H CRCs were collected by a review of medical records and microscopic examination of H\&E-stained FFPE tissue slides. Age, gender, tumour location and TNM stage (American Joint Committee on Cancer/Union for International Cancer Control cancer stage 7th edition) were included as clinical parameters. Mean age of the $212 \mathrm{MSI}-\mathrm{H}$ CRC patients was 58 years (ranging from 20 years to 95 years). Morphological tumour grade (WHO three-tier grade), mucinous component, medullary component, signet ring cell component, tumour border, tumour budding, lymphovascular invasion and perineural invasion were examined as histopathological parameters by three pathologists (JHK, JMB and GHK). Times of death, cancer recurrence and the last clinical follow-up were investigated as disease-free survival (DFS) data of 212 patients with MSI-H CRC. DFS was defined as the time from surgery to death or cancer recurrence, or to the last clinical follow-up. The median follow-up time was 1811 days (ranging from 29 to 3261 days). Patient death or CRC recurrence was used for the end-point of DFS-based survival analysis. In this study, the number of death or cancer recurrence observed in the 212 patients with MSI-H CRC was 49.

\section{Molecular data}

Immunostaining for mismatch repair proteins, including MLH1, MSH2, PMS2 and MSH6, in FFPE tissues of 212 MSI-H CRCs and DNA analyses to determine the $\mathrm{CpG}$ island methylator phenotype status and KRAS/BRAF mutations in genomic DNA samples extracted from 212 MSI-H CRC tissues were performed as previously described. ${ }^{12}$ In this study, of the 212 MSI-H CRCs, KRAS mutation analysis was conducted in 205 cases because insufficient DNA was isolated from seven tissue samples.

\section{Statistical analysis}

IBM SPSS statistics V.20 (Chicago, Illinois, USA) was used for all the statistical analyses in this study. To evaluate interobserver agreement of categorical data by each CLR criteria, $\kappa$ values by Cohen's $\kappa$ statistics were calculated. To determine interobserver agreement of continuous data (CLR density and the largest LA size), the intraclass correlation coefficients (ICC) were calculated. For comparisons of the categorical variables, the $\chi^{2}$ test or Fisher's exact test was performed. The Kaplan-Meier method with a log-rank test was performed for univariate survival analysis using DFS data of our study samples. To identify independent prognostic factors, the Cox proportional hazards regression model-based multivariable analysis was conducted. Cox multivariable analysis was performed using parameters determined to be significant $(p<0.05)$ in the univariable Kaplan-Meier survival analysis. The log-minus-log plot was used to verify the proportional hazards assumption of the Cox model. All $\mathrm{p}$ values were two-sided, and $\mathrm{p}<0.05$ determined the statistical significance.

\section{RESULTS}

\section{Interobserver reproducibility of CLR assessment criteria}

In Cohen's $\kappa$ statistics using final categorised data of CLR assessment results in 212 MSI-H CRCs evaluated by two experienced gastrointestinal pathologists (JHK and K-JK), CLR assessment using Väyrynen-Mäkinen criteria showed the best interobserver agreement (agreement rate, $87 \%$; $\kappa$ value, $0.71 ; \mathrm{p}<0.001$; table 1 ), followed by CLR assessment using Ueno criteria (agreement rate, $80 \%$; $\kappa$ value, $0.56 ; \mathrm{p}<0.001$; table 1 ) and CLR assessment using Graham-Appelman criteria (agreement rate, $78 \%$; $\kappa$ value, 0.47; $\mathrm{p}<0.001$; table 1 ). The $\kappa$ values of the Väyrynen-Mäkinen criteriabased assessment (0.71) and the Ueno criteria-based assessment (0.56) indicate a substantial degree and moderate degree of interobserver agreement, respectively. ${ }^{13}$ ICC analyses using raw continuous data of CLR density and the largest LA size demonstrated moderate to good correlations between the two pathologists (ICC, $0.76 ; 95 \%$ CI 0.7 to 0.81 for CLR density and ICC, $0.58 ; 95 \%$ CI 0.48 to 0.66 for the largest LA size; table 1 ).

\section{Clinicopathological and molecular associations of CLR assessment criteria}

Differential clinical, pathological and molecular features of MSI-H CRCs depending on CLR status determined by Ueno criteria, Väyrynen-Mäkinen criteria or Graham-Appelman criteria are summarised in table 2 . In the results of the association analysis using Ueno criteria-based CLR classification, active CLR (largest LA size $\geq 1 \mathrm{~mm}$ ) was significantly associated with early stage (stage I/II; $\mathrm{p}=0.019$ ), tumour-budding negativity $(\mathrm{p}=0.018)$ and the absence of lymphovascular invasion $(\mathrm{p}=0.008)$ (table 2). In the analysis using Väyrynen-Mäkinen 

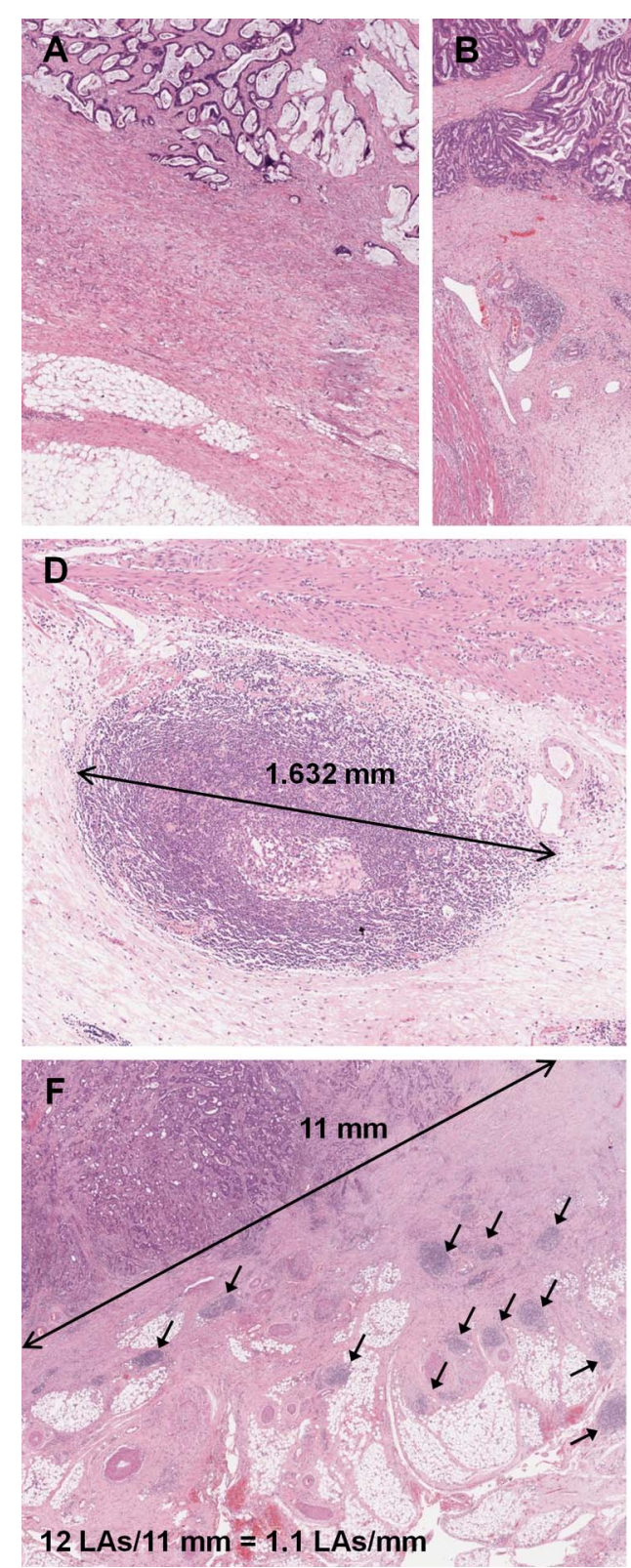
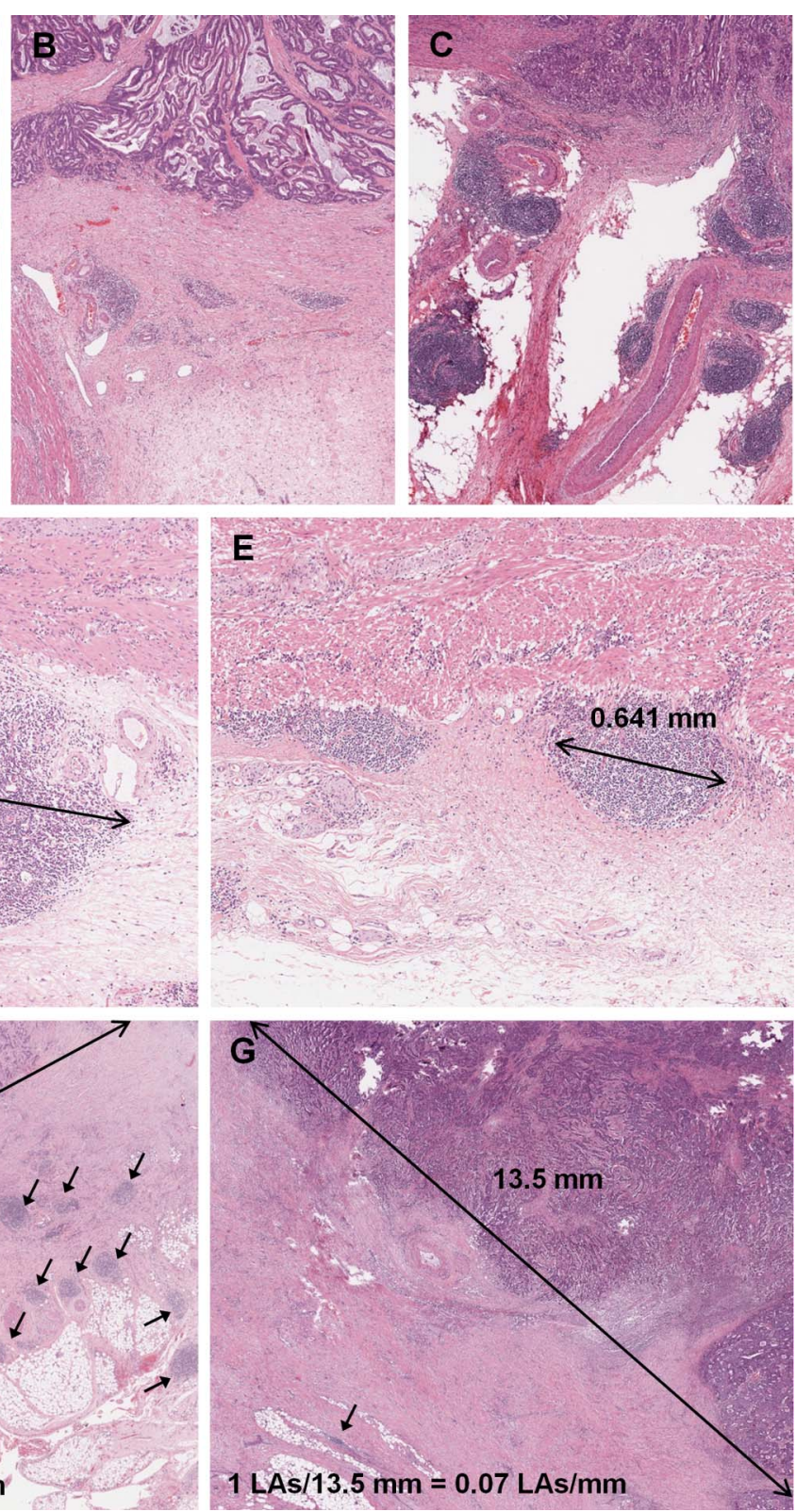

Figure 1 Histopathological assessment of CLR status in microsatellite instability-high colorectal carcinomas. (A-C) Representative histology of grades 0 (A), 1 (B) and 2 (C) CLR statuses determined by Graham-Appelman criteria (H\&E, $\times 20$ ). (D-E) Representative histology of active (D) and inactive (E) CLR statuses determined by Ueno criteria (H\&E, $\times 50)$. (F-G) Representative histology of high-density (F) and low-density (G) CLR statuses determined by Väyrynen-Mäkinen criteria (H\&E, ×10). CLR, Crohn-like lymphoid reaction; LA, lymphoid aggregate.

criteria, high-density CLR $(\geq 0.38 \mathrm{LAs} / \mathrm{mm})$ was significantly correlated with early stage (stage I/II; $\mathrm{p}=0.03$ ) and low tumour grade (G1/G2; $\mathrm{p}=0.022$ ) (table 2). In the analysis using Graham-Appelman criteria, intense CLR (grade 2) was significantly related to early stage (stage $\mathrm{I} / \mathrm{II} ; \mathrm{p}=0.048$ ) (table 2 ).

\section{Prognostic value of CLR assessment criteria}

In the Kaplan-Meier survival analysis with log-rank test, patients with active CLR $(\geq 1 \mathrm{~mm})$ showed a significantly better DFS than those with inactive CLR $(<1 \mathrm{~mm})$ in 212 patients with MSI-H CRC $(p=0.005$; figure $2 \mathrm{~A})$. There was also an

Table 1 Comparison of interobserver reproducibility of the three Crohn-like lymphoid reaction assessment criteria

\begin{tabular}{llll}
\hline Values & Ueno criteria & Väyrynen-Mäkinen criteria & Graham-Appelman criteria \\
\hline Agreement rate $(\%)$ & 80 & 87 & 78 \\
$\kappa$ value ( $p$ value) & $0.56(<0.001)$ & $0.71(<0.001)$ & $0.47(<0.001)$ \\
Intraclass correlation coefficient $(95 \% \mathrm{Cl})$ & $0.58(0.48$ to 0.66$)$ & $0.76(0.7$ to 0.81$)$ & NA \\
\hline $\mathrm{NA}$ not applicable & &
\end{tabular}


Table 2 Clinicopathological and molecular characteristics according to CLR status determined by the three assessment criteria in MSI-H CRCs $(\mathrm{n}=212)$

\begin{tabular}{|c|c|c|c|c|c|c|c|c|c|c|}
\hline \multirow[b]{2}{*}{ Characteristics } & \multirow[b]{2}{*}{ Total cases } & \multicolumn{3}{|c|}{ Ueno criteria } & \multicolumn{3}{|c|}{ Väyrynen-Mäkinen criteria } & \multicolumn{3}{|c|}{ Graham-Appelman criteria } \\
\hline & & $\begin{array}{l}\text { Active CLR } \\
(\geq 1 \mathrm{~mm}) \\
\text { cases }(\%)\end{array}$ & $\begin{array}{l}\text { Inactive CLR } \\
(<1 \mathrm{~mm}) \\
\text { cases }(\%)\end{array}$ & $\mathrm{p}$ Value & $\begin{array}{l}\text { High-density CLR } \\
(\geq 0.38 \mathrm{LAs} / \mathrm{mm}) \\
\text { cases }(\%)\end{array}$ & $\begin{array}{l}\text { Low-density CLR } \\
(<0.38 \mathrm{LAs} / \mathrm{mm}) \\
\text { cases }(\%)\end{array}$ & $\mathrm{p}$ Value & $\begin{array}{l}\text { Intense CLR } \\
\text { (grade 2) } \\
\text { cases (\%) }\end{array}$ & $\begin{array}{l}\text { No or mild } \\
\text { CLR (grade 0/1) } \\
\text { cases (\%) }\end{array}$ & $\mathrm{p}$ Value \\
\hline \multicolumn{11}{|l|}{ Age (years) } \\
\hline$<58$ & 101 & $43(50)$ & $58(46)$ & \multirow[t]{2}{*}{0.57} & $66(48)$ & $35(47)$ & \multirow[t]{2}{*}{0.941} & $22(46)$ & $79(48)$ & \multirow[t]{2}{*}{0.776} \\
\hline$\geq 58$ & 111 & $43(50)$ & $68(54)$ & & $72(52)$ & $39(53)$ & & $26(54)$ & $85(52)$ & \\
\hline \multicolumn{11}{|l|}{ Gender } \\
\hline Male & 112 & $43(50)$ & $57(45)$ & \multirow[t]{2}{*}{0.495} & 77 (56) & 35 (47) & \multirow[t]{2}{*}{0.237} & $30(63)$ & $82(50)$ & \multirow[t]{2}{*}{0.127} \\
\hline Female & 100 & $43(50)$ & $69(55)$ & & $61(44)$ & $39(53)$ & & $18(38)$ & $82(50)$ & \\
\hline \multicolumn{11}{|l|}{ Tumour location } \\
\hline Proximal & 137 & $59(69)$ & $78(62)$ & \multirow[t]{2}{*}{0.316} & $93(67)$ & $44(59)$ & \multirow[t]{2}{*}{0.25} & $36(75)$ & $101(62)$ & 0.087 \\
\hline Distal & 75 & $27(31)$ & $48(38)$ & & $45(33)$ & $30(41)$ & & $12(25)$ & $63(38)$ & \\
\hline AJCC/UICC TNM & stage & & & & & & & & & \\
\hline Stage I/II & 138 & $64(74)$ & $74(59)$ & 0.019 & $97(70)$ & $41(55)$ & 0.03 & $37(77)$ & $101(62)$ & 0.048 \\
\hline Stage III/IV & 74 & $22(26)$ & $52(41)$ & & $41(30)$ & $33(45)$ & & $11(23)$ & $63(38)$ & \\
\hline WHO tumour gra & & & & & & & & & & \\
\hline $\mathrm{G} 1 / \mathrm{G} 2$ & 170 & $71(83)$ & $99(79)$ & 0.475 & $117(85)$ & $53(72)$ & 0.022 & $39(81)$ & $131(80)$ & 0.834 \\
\hline G3 & 42 & $15(17)$ & $27(21)$ & & 21 (15) & $21(28)$ & & $9(19)$ & $33(20)$ & \\
\hline Mucinous compo & nent & & & & & & & & & \\
\hline Absent & 90 & $36(42)$ & $54(43)$ & 0.885 & $60(43)$ & $30(41)$ & 0.68 & $24(50)$ & $66(40)$ & 0.229 \\
\hline Present & 122 & $50(58)$ & $72(57)$ & & 78 (57) & $44(59)$ & & $24(50)$ & $98(60)$ & \\
\hline Medullary compc & nent & & & & & & & & & \\
\hline Absent & 205 & $82(95)$ & $123(98)$ & 0.445 & $133(96)$ & $72(97)$ & 1 & 47 (98) & $158(96)$ & 1 \\
\hline Present & 7 & $4(5)$ & $3(2)$ & & $5(4)$ & $2(3)$ & & $1(2)$ & $6(4)$ & \\
\hline Signet ring cell c & omponent & & & & & & & & & \\
\hline Absent & 192 & $79(92)$ & $113(90)$ & 0.594 & $124(90)$ & $68(92)$ & 0.629 & $45(94)$ & $147(90)$ & 0.576 \\
\hline Present & 20 & 7 (8) & $13(10)$ & & $14(10)$ & $6(8)$ & & $3(6)$ & $17(10)$ & \\
\hline Tumour border & & & & & & & & & & \\
\hline Expanding & 34 & $16(19)$ & $18(14)$ & 0.4 & $24(17)$ & $10(14)$ & 0.463 & $11(23)$ & $23(14)$ & 0.14 \\
\hline Infiltrative & 178 & $70(81)$ & $108(86)$ & & $114(83)$ & $64(86)$ & & 37 (77) & $141(86)$ & \\
\hline Tumour budding & & & & & & & & & & \\
\hline Negative & 168 & $75(87)$ & $93(74)$ & 0.018 & $112(81)$ & $56(76)$ & 0.348 & $39(81)$ & $129(79)$ & 0.697 \\
\hline Positive & 44 & $11(13)$ & $33(26)$ & & $26(19)$ & $18(24)$ & & $9(19)$ & $35(21)$ & \\
\hline Lymphovascular & nvasion & & & & & & & & & \\
\hline Absent & 157 & $72(84)$ & $85(67)$ & 0.008 & $108(78)$ & $49(66)$ & 0.057 & $39(81)$ & $118(72)$ & 0.196 \\
\hline Present & 55 & $14(16)$ & $41(33)$ & & $30(22)$ & $25(34)$ & & $9(19)$ & $46(28)$ & \\
\hline Perineural invasi & & & & & & & & & & \\
\hline Absent & 196 & $82(95)$ & $114(90)$ & 0.187 & $131(95)$ & $65(88)$ & 0.062 & $45(94)$ & $151(92)$ & 1 \\
\hline Present & 16 & $4(5)$ & $12(10)$ & & $7(5)$ & $9(12)$ & & $3(6)$ & $13(8)$ & \\
\hline MLH1 expression & & & & & & & & & & \\
\hline Loss & 134 & $57(66)$ & $77(61)$ & 0.444 & $89(64)$ & $45(61)$ & 0.596 & $32(67)$ & $102(62)$ & 0.572 \\
\hline Retained & 78 & $29(34)$ & $49(39)$ & & $49(36)$ & $29(39)$ & & $16(33)$ & $62(38)$ & \\
\hline MSH2 expression & & & & & & & & & & \\
\hline Loss & 66 & $26(30)$ & $40(32)$ & 0.815 & $41(30)$ & $25(34)$ & 0.541 & $12(25)$ & $54(33)$ & 0.297 \\
\hline Retained & 146 & $60(70)$ & $86(68)$ & & $97(70)$ & $49(66)$ & & $36(75)$ & $110(67)$ & \\
\hline PMS2 expression & & & & & & & & & & \\
\hline Loss & 141 & $60(70)$ & $81(64)$ & 0.406 & $94(68)$ & $47(64)$ & 0.499 & $35(73)$ & $106(65)$ & 0.285 \\
\hline Retained & 71 & $26(30)$ & $45(36)$ & & $44(32)$ & 27 (36) & & $13(27)$ & $58(35)$ & \\
\hline MSH6 expression & & & & & & & & & & \\
\hline Loss & 73 & $27(31)$ & $46(37)$ & 0.442 & $44(32)$ & $29(39)$ & 0.286 & $13(27)$ & $60(37)$ & 0.223 \\
\hline Retained & 139 & $59(69)$ & $80(63)$ & & $94(68)$ & $45(61)$ & & $35(73)$ & $104(63)$ & \\
\hline KRAS mutation & & & & & & & & & & \\
\hline Wild type & 163 & $68(81)$ & $95(79)$ & 0.67 & $103(77)$ & $60(83)$ & 0.319 & $33(72)$ & $130(82)$ & 0.138 \\
\hline Mutant & 42 & $16(19)$ & $26(21)$ & & $30(23)$ & $12(17)$ & & $13(28)$ & $29(18)$ & \\
\hline BRAF mutation & & & & & & & & & & \\
\hline Wild type & 188 & $77(90)$ & $111(88)$ & 0.745 & $124(90)$ & $64(86)$ & 0.461 & $43(90)$ & $145(88)$ & 0.822 \\
\hline Mutant & 24 & $9(10)$ & $15(12)$ & & $14(10)$ & $10(14)$ & & $5(10)$ & $19(12)$ & \\
\hline
\end{tabular}




\section{Original article}

Table 2 Continued

\begin{tabular}{|c|c|c|c|c|c|c|c|c|c|c|}
\hline \multirow[b]{2}{*}{ Characteristics } & \multirow[b]{2}{*}{ Total cases } & \multicolumn{3}{|c|}{ Ueno criteria } & \multicolumn{3}{|c|}{ Väyrynen-Mäkinen criteria } & \multicolumn{3}{|c|}{ Graham-Appelman criteria } \\
\hline & & $\begin{array}{l}\text { Active CLR } \\
(\geq 1 \mathrm{~mm}) \\
\text { cases (\%) }\end{array}$ & $\begin{array}{l}\text { Inactive CLR } \\
(<1 \mathrm{~mm}) \\
\text { cases }(\%)\end{array}$ & $\mathrm{p}$ Value & $\begin{array}{l}\text { High-density CLR } \\
(\geq 0.38 \mathrm{LAs} / \mathrm{mm}) \\
\text { cases }(\%)\end{array}$ & $\begin{array}{l}\text { Low-density CLR } \\
(<0.38 \mathrm{LAs} / \mathrm{mm}) \\
\text { cases }(\%)\end{array}$ & $\mathrm{p}$ Value & $\begin{array}{l}\text { Intense CLR } \\
\text { (grade 2) } \\
\text { cases (\%) }\end{array}$ & $\begin{array}{l}\text { No or mild } \\
\text { CLR (grade 0/1) } \\
\text { cases (\%) }\end{array}$ & p Value \\
\hline \multicolumn{11}{|l|}{ CIMP status } \\
\hline CIMP-H & 54 & $19(22)$ & $35(28)$ & 0.351 & $31(22)$ & $23(31)$ & 0.17 & $8(17)$ & $46(28)$ & 0.111 \\
\hline CIMP-L/0 & 158 & 67 (78) & $91(72)$ & & $107(78)$ & 51 (69) & & $40(83)$ & $118(72)$ & \\
\hline
\end{tabular}

CLR, Crohn-like lymphoid reaction; MSI-H, microsatellite instability-high; CRC, colorectal cancer; CIMP, CpG island methylator phenotype; CIMP-H, CIMP-high; CIMP-L/0, CIMP-low or CIMP-negative; LA, lymphoid aggregate; AJCC/UICC, American Joint Committee on Cancer/Union for International Cancer Control.

obvious difference in DFS between patient subgroups classified by Väyrynen-Mäkinen criteria $(p=0.001$; figure 2B). However, Graham-Appelman criteria-based patient subgroups failed to demonstrate a significant survival difference although there was a tendency towards favourable survival of the patient subgroup with intense CLR (grade 2) $(\mathrm{p}=0.326$; figure $2 \mathrm{C}$ ). In the multivariable survival analysis using the Cox proportional hazards regression model, both Ueno criteria-based CLR activity classification and Väyrynen-Mäkinen criteria-based CLR density classification were determined to be independent prognostic factors in MSI-H CRCs (HR, 0.47; 95\% CI 0.24 to 0.9 ; $\mathrm{p}=0.024$ for Ueno criteria and HR, $0.5 ; 95 \%$ CI 0.28 to $0.89 ; \mathrm{p}=0.017$ for Väyrynen-Mäkinen criteria) (tables 3 and 4).

\section{DISCUSSION}

Although CLR acquired its unique name based on a histological resemblance to Crohn's disease, ${ }^{1}$ CLR can be regarded as induced ectopic lymphoid-like structures or tertiary lymphoid organs associated with adaptive host immune responses against tumours in CRC. ${ }^{5}{ }^{14}$ Therefore, previous studies have consistently revealed the favourable prognostic effect of CLR in patients with CRC. ${ }^{1-5}$ However, there has been a lack of consensus on optimal histological criteria for CLR assessment in CRC. Until recently, a traditional three-tier grading system suggested by Graham and Appelman has been used unquestioningly to evaluate CLR in CRC, but this assessment method has some limitations originating from its semiquantitative, subjective
Figure 2 Kaplan-Meier survival curves by CLR status in 212 patients with microsatellite instability-high colorectal carcinoma. (A) Ueno criteria-based CLR status. (B) Väyrynen-Mäkinen criteria-based CLR status. (C) Graham-Appelman criteria-based CLR status. CLR, Crohn-like lymphoid reaction; LA, lymphoid aggregate.
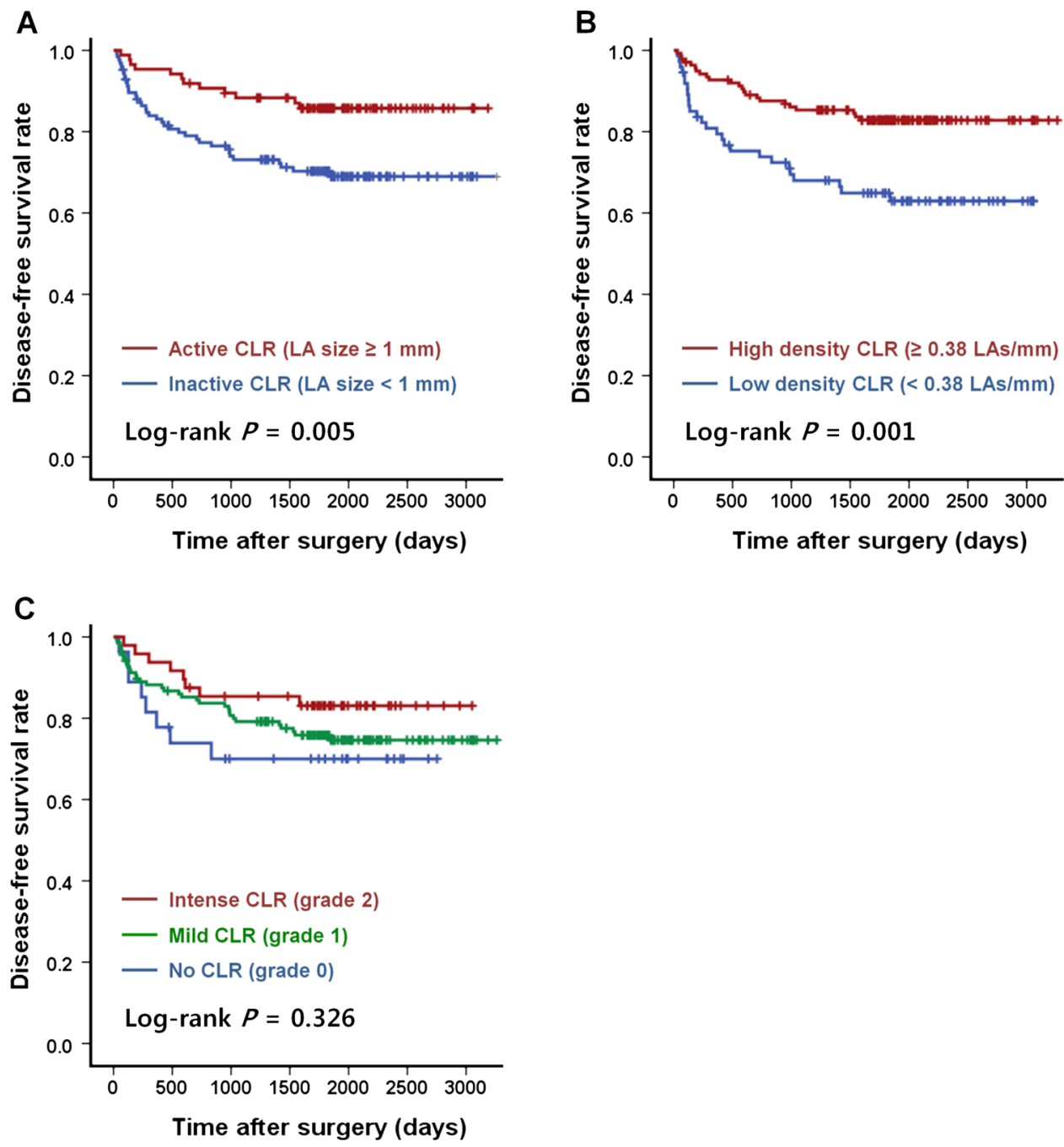
Table 3 Multivariable survival analysis by Cox proportional hazards regression model: Ueno criteria for CLR variable

\begin{tabular}{|c|c|c|c|}
\hline Variables & Case No & $\mathrm{HR}(95 \% \mathrm{Cl})$ & p Value \\
\hline \multicolumn{4}{|l|}{ Ueno criteria-based CLR status } \\
\hline Inactive (largest LA size $<1 \mathrm{~mm}$ ) & 126 & Reference & \\
\hline Active (largest LA size $\geq 1 \mathrm{~mm}$ ) & 86 & $0.47(0.24$ to 0.9$)$ & 0.024 \\
\hline \multicolumn{4}{|l|}{ AJCC/UICC TNM stage } \\
\hline Stage I/II & 138 & Reference & \\
\hline Stage III/IV & 74 & 3.61 (1.96 to 6.67$)$ & $<0.001$ \\
\hline \multicolumn{4}{|l|}{ WHO tumour grade } \\
\hline G1/G2 & 170 & Reference & \\
\hline G3 & 42 & 1.86 (1.01 to 3.41$)$ & 0.046 \\
\hline \multicolumn{4}{|l|}{ Age (years) } \\
\hline$<58$ & 101 & Reference & \\
\hline$\geq 58$ & 111 & $1.39(0.72$ to 2.7$)$ & 0.332 \\
\hline \multicolumn{4}{|l|}{ CIMP status } \\
\hline CIMP-L/O & 158 & Reference & \\
\hline CIMP-H & 54 & $1.38(0.71$ to 2.7$)$ & 0.347 \\
\hline
\end{tabular}

nature. Notably, two independent studies conducted by the Ueno group and the Väyrynen-Mäkinen group recently suggested novel criteria for CLR assessment, which are LA sizebased and LA density-based methods, respectively. ${ }^{4} 5$ Though each study concluded that each assessment criteria had strengths in terms of objectivity and prognostication, there has been no external validation of these novel criteria in CRC. In this regard, our study provides timely data regarding the comparison of interobserver reproducibility and prognostic value between CLR assessment criteria, including Graham-Appelman criteria, Ueno criteria and Väyrynen-Mäkinen criteria, in CRC.

To clarify prognostic significance of the CLR assessment method itself, the exclusion of the prognostic effect of MSI-H is important because MSI-H is a well-known prognostic

Table 4 Multivariable survival analysis by Cox proportional hazards regression model: Väyrynen-Mäkinen criteria for CLR variable

\begin{tabular}{|c|c|c|c|}
\hline Variables & Case No & HR $(95 \% \mathrm{Cl})$ & p Value \\
\hline \multicolumn{4}{|c|}{ Väyrynen-Mäkinen criteria-based CLR status } \\
\hline Low density (<0.38 LAs/mm) & 74 & Reference & \\
\hline High density ( $\geq 0.38 \mathrm{LAs} / \mathrm{mm})$ & 138 & $0.5(0.28$ to 0.89$)$ & 0.017 \\
\hline \multicolumn{4}{|l|}{ AJCC/UICC TNM stage } \\
\hline Stage $I / I I$ & 138 & Reference & \\
\hline Stage III/IV & 74 & 3.68 (1.98 to 6.82$)$ & $<0.001$ \\
\hline \multicolumn{4}{|l|}{ WHO tumour grade } \\
\hline G1/G2 & 170 & Reference & \\
\hline G3 & 42 & $1.63(0.89$ to 3$)$ & 0.117 \\
\hline \multicolumn{4}{|l|}{ Age (years) } \\
\hline$<58$ & 101 & Reference & \\
\hline$\geq 58$ & 111 & 1.51 (0.78 to 2.92$)$ & 0.227 \\
\hline \multicolumn{4}{|l|}{ CIMP status } \\
\hline CIMP-L/0 & 158 & Reference & \\
\hline CIMP-H & 54 & $1.34(0.69$ to 2.61$)$ & 0.39 \\
\hline
\end{tabular}

molecular factor in CRC. Therefore, in our study, by using a cohort consisting of a large series of MSI-H CRC, our data successfully revealed that CLR activity assessed by Ueno criteria and CLR density assessed by Väyrynen-Mäkinen criteria can be independent prognostic factors in CRC regardless of the favourable prognostic effect of MSI-H (tables 3 and 4). Clinicopathologically, it was also identified that active CLR by Ueno criteria, high-density CLR by Väyrynen-Mäkinen criteria and intense CLR by Graham-Appelman criteria were significantly associated with early cancer stage (stage I/II) (table 2). Regarding this finding, two interpretations can be suggested: (1) CLR may play an active role in attenuating the invasive and metastatic potential of tumours; (2) CLR may develop prominently in innately less aggressive tumours. Both these interpretations can support the favourable prognostic effect of CLR in CRC.

In conclusion, two novel quantitative criteria, Ueno criteria and Väyrynen-Mäkinen criteria, are confirmed to be useful and reliable methods for CLR assessment in CRC. It is strongly anticipated that a cancer staging system based on host immune responses against tumours will be developed in the near future. $^{15}$ CLR activity and CLR density will be able to be included as important components of the immune response staging system for CRC prognostication.

\section{Take home messages}

- Crohn-like lymphoid reaction (CLR) has been investigated as a histological prognosticator in colorectal carcinoma (CRC), but a consensus on optimal assessment criteria for CLR has not been reached.

- Recently, two quantitative criteria for CLR assessment have been suggested by the Ueno group and the VäyrynenMäkinen group, which are based on the measurement of the largest lymphoid aggregate (LA) size and LA density, respectively.

- Our study reveals that both Ueno criteria and VäyrynenMäkinen criteria can be used as useful tools for CLR assessment in CRC in terms of reproducibility and prognostic value.

Contributors JHK designed the study, performed histopathological and statistical analyses, and drafted the manuscript. K-JK and JMB performed the histopathological assessment. Y-YR and HSL conducted the collection of tissue samples and clinicopathological data. N-YC performed molecular experiments. GHK supervised the study, reviewed histopathological and molecular data and revised the manuscript. All the authors approved the final version of the manuscript.

Funding This study was supported by a grant from Basic Science Research Program through the National Research Foundation (NRF) funded by the Ministry of Education (2013R1A1A2059080), a grant from the Korean Health Technology R\&D Project, Ministry of Health \& Welfare (HI13C1804), Priority Research Centres Program through the NRF funded by the Ministry of Education, Science and Technology (2009-0093820), and the NRF grant funded by the Ministry of Science, ICT, and Future Planning (2011-0030049).

\section{Competing interests None.}

Ethics approval Institutional Review Board, Seoul National University Hospital Seoul, Korea (SNUH IRB No. H-1203-072-402).

Provenance and peer review Not commissioned; externally peer reviewed.

\section{REFERENCES}

1 Graham DM, Appelman HD. Crohn's-like lymphoid reaction and colorectal carcinoma: a potential histologic prognosticator. Mod Pathol 1990;3:332-5. 


\section{Original article}

2 Harrison JC, Dean PJ, el-Zeky F, et al. Impact of the Crohn's-like lymphoid reaction on staging of right-sided colon cancer: results of multivariate analysis. Hum Pathol 1995;26:31-8.

3 Ogino S, Nosho K, Irahara N, et al. Lymphocytic reaction to colorectal cancer is associated with longer survival, independent of lymph node count, microsatellite instability, and CpG island methylator phenotype. Clin Cancer Res 2009;15:6412-20.

4 Ueno $\mathrm{H}$, Hashiguchi $Y$, Shimazaki $\mathrm{H}$, et al. Objective criteria for crohn-like lymphoid reaction in colorectal cancer. Am J Clin Pathol 2013;139:434-41.

5 Vayrynen JP, Sajanti SA, Klintrup K, et al. Characteristics and significance of colorectal cancer associated lymphoid reaction. Int J Cancer 2014;134:2126-35.

6 Alexander J, Watanabe T, Wu TT, et al. Histopathological identification of colon cancer with microsatellite instability. Am J Pathol 2001;158:527-35.

7 Buckowitz A, Knaebel HP, Benner A, et al. Microsatellite instability in colorectal cancer is associated with local lymphocyte infiltration and low frequency of distant metastases. Br J Cancer 2005;92:1746-53.

8 Greenson JK, Huang SC, Herron C, et al. Pathologic predictors of microsatellite instability in colorectal cancer. Am J Surg Pathol 2009;33:126-33.

9 Popat $S$, Hubner R, Houlston RS. Systematic review of microsatellite instability and colorectal cancer prognosis. J Clin Oncol 2005;23:609-18.
10 Guastadisegni C, Colafranceschi M, Ottini L, et al. Microsatellite instability as a marker of prognosis and response to therapy: a meta-analysis of colorectal cancer survival data. Eur J Cancer 2010;46:2788-98.

11 Boland CR, Thibodeau SN, Hamilton SR, et al. A National Cancer Institute Workshop on Microsatellite Instability for cancer detection and familial predisposition: development of international criteria for the determination of microsatellite instability in colorectal cancer. Cancer Res 1998;58:5248-57.

$12 \mathrm{Kim} \mathrm{JH}$, Kim KJ, Rhee YY, et al. Expression status of wild-type HSP110 correlates with HSP110 $\mathrm{T}_{17}$ deletion size and patient prognosis in microsatellite-unstable colorectal cancer. Mod Pathol 2014;27:443-53.

13 Landis JR, Koch GG. The measurement of observer agreement for categorical data. Biometrics 1977;33:159-74.

14 Coppola D, Nebozhyn M, Khalil F, et al. Unique ectopic lymph node-like structures present in human primary colorectal carcinoma are identified by immune gene array profiling. Am J Pathol 2011;179:37-45.

15 Galon J, Mlecnik B, Bindea G, et al. Towards the introduction of the 'Immunoscore' in the classification of malignant tumours. J Pathol 2014:232:199-209. 